\title{
BMJ Open PrEP distribution in pharmacies: a systematic review
}

\author{
Caitlin E Kennedy, ${ }^{1}$ Ping Teresa Yeh (D) , ${ }^{1}$ Kaitlyn Atkins, ${ }^{1}$ Laura Ferguson, ${ }^{2}$ \\ Rachel Baggaley, ${ }^{3}$ Manjulaa Narasimhan ${ }^{4}$
}

To cite: Kennedy CE, Yeh PT, Atkins K, et al. PrEP distribution in pharmacies: a systematic review. BMJ Open 2022;12:e054121. doi:10.1136/ bmjopen-2021-054121

- Prepublication history and additional supplemental material for this paper are available online. To view these files, please visit the journal online (http://dx.doi.org/10.1136/ bmjopen-2021-054121).

Received 03 June 2021 Accepted 28 January 2022

Check for updates

(c) Author(s) (or their employer(s)) 2022. Re-use permitted under CC BY-NC. No commercial re-use. See rights and permissions. Published by BMJ.

${ }^{1}$ Department of International Health, Johns Hopkins University Bloomberg School of Public Health, Baltimore, Maryland, USA

${ }^{2}$ Keck School of Medicine, Institute on Inequalities in Global Health, University of Southern California, Los Angeles,

California, USA

${ }^{3}$ Department of HIV, Viral

Hepatitis, and STIs, World

Health Organization, Geneva,

Switzerland

${ }^{4}$ Department of Sexual and

Reproductive Health and

Research, World Health

Organization, Geneva,

Switzerland

Correspondence to

Dr Manjulaa Narasimhan;

narasimhanm@who.int

\section{ABSTRACT}

Introduction Novel mechanisms of service delivery are needed to expand access to pre-exposure prophylaxis (PrEP) for HIV prevention. Providing PrEP directly through pharmacies could offer an additional option for reaching potential users.

Methods We conducted a systematic review of studies examining effectiveness, values and preferences of end users and health workers, and cost of PrEP initiation and continuation through pharmacies (pharmacy access). We searched PubMed, CINAHL, LILACS and EMBASE through 2 December 2020. We also searched clinical trial registries and recent HIV conference abstracts. Standardised methods were used to search, screen and extract data from included studies.

Results No studies met the inclusion criteria for the effectiveness review, for either PrEP initiation or continuation. However, six 'case studies' presenting non-comparative data from PrEP pharmacy programmes demonstrated feasibility of this model in the USA. Eleven studies reported values and preferences of end users and health workers. In the USA, Kenya and South Africa, potential PrEP clients generally supported pharmacy access, although some preferred clinics. One study of PrEP pharmacy clients found all would 'definitely recommend' the programme. Six studies found pharmacists were generally supportive of offering PrEP; one study including doctors found more limited favour, while one study of diverse Kenyan stakeholders found broad support. Three studies reported cost data indicating client willingness to pay in the USA and Kenya and initial sustainability of a clinic financial model in the USA.

Conclusion Provision of PrEP through pharmacies has been demonstrated to be feasible in the USA and acceptable to potential end users and stakeholders in multiple settings. Limited evidence on effectiveness and requirements for laboratory testing and assurance of highquality services may limit enthusiasm for this approach. Further research is needed to determine if pharmacy access is a safe and effective way to help achieve global HIV prevention goals.

PROSPERO registration number CRD42021231650.

\section{INTRODUCTION}

HIV pre-exposure prophylaxis (PrEP) is the use of antiretroviral drugs by HIV-uninfected individuals to prevent HIV infection. PrEP may either be taken orally in a daily pill (generally containing tenofovir plus emtricitabine, or
Strengths and limitations of this study

This systematic review used a comprehensive search for articles on the effectiveness of preexposure prophylaxis distribution through pharmacies, and on costs of this model and values and preferences of end users and health workers.

- Because this is a rapidly growing field, we may have missed new publications or articles which used terms which were not in our search strategy.

- The generalisability of our findings globally may be limited, since nearly all evidence included in our review came from the USA besides a few studies from Kenya and South Africa.

TDF/FTC), event-driven (at the time of sex) or in the form of a dapivirine vaginal ring; recent data suggest that long-acting injectable PrEP may soon be an additional option. However, not all forms of PrEP are available in all settings globally; in most low-income countries, only daily oral PrEP is available. WHO recommends that people at substantial risk of HIV infection should be offered PrEP as an additional prevention choice as part of a combination prevention approach, ${ }^{1}$ which includes integration of sexual and reproductive health (SRH), HIV and sexually transmitted infection (STI) services. ${ }^{2}$

Novel approaches to service delivery are being developed to expand PrEP access. Within clinical services, PrEP has been provided through community health clinics, sexually transmitted disease clinics and primary care providers. ${ }^{3}$ Community health workers have been trained to conduct PrEP outreach and provide referrals to PrEP prescription services. ${ }^{4}$ There are also mobile applications that offer PrEP prescriptions from a qualified health worker but without an in-person visit. ${ }^{5}$ Making PrEP available outside of formal health facilities has the potential to reduce barriers to access, improve autonomy and increase use and coverage of these effective HIV prevention options. It also may be a way to reach people 
who could benefit from PrEP but do not feel comfortable attending a clinic.

Pharmacies have been described as one area of untapped potential for PrEP delivery. ${ }^{6-8}$ Pharmacies are often more accessible than health facilities, as they are usually conveniently located within communities, may have longer hours (including nights and weekends) and are available without an appointment. They also serve a wide range of health issues, so may reduce stigma associated with seeking HIV-related services. However, writing or filling PrEP prescriptions is not within pharmacists' scope of practice in many settings, so considering expansion of PrEP to pharmacies must be done with consideration of local regulatory guidelines.

This systematic review evaluates the evidence for distributing PrEP through pharmacies. We conducted this systematic review in the context of expanding the evidence base of WHO's normative guidance on self-care interventions. ${ }^{9}$ This guidance includes recommendations for over-the-counter pharmacy access to oral contraceptives as a means to expand access and coverage and is linked to WHO's competency-based training of pharmacists. ${ }^{10}$ This review is also being conducted in response to the COVID-19 pandemic that has seen overstretched health systems and closures of medical facilities due to country-wide lockdowns globally ${ }^{11}$ and where multimonth prescribing, including for clients initiating PrEP, ${ }^{12}$ has been prioritised by WHO where appropriate.

\section{METHODS}

This review addressed two related questions: whether PrEP initiation should happen in pharmacies, and whether PrEP continuation should happen in pharmacies. We focused on in-person pharmacy initation and continuation, and excluded telemedicine-based approaches. We reviewed the extant literature in three areas relevant to answering these questions: effectiveness of the intervention, values and preferences of end users and health workers and cost information. The review followed Preferred Reporting Items for Systematic Reviews and Meta-Analyses guidelines. ${ }^{13}$

\section{Effectiveness review}

PICO question 1-initiation

Should PrEP initiation be available following screening by a pharmacist, without a prescription?

Population: individuals interested in PrEP.

Intervention: PrEP access through a pharmacy without a prescription by a health worker (defined as a nonpharmacist health worker).

Comparator: PrEP access by prescription from a health worker.

Outcomes:

1. Uptake of PrEP (initial use).

2. Continuation of PrEP (continued use or an intermittent pattern of use related to risk exposure).
3. Correct use of PrEP (either daily or event-driven), including stopping and starting.

4. HIV acquisition/incidence.

5. Side effects, adverse events and clinical harms (renal disease, STI acquisition, STI treatment).

6. Uptake of regular HIV testing (1 month after initiation and 3 monthly thereafter while taking PrEP-or if taking PrEP intermittently (seasons of risk), prior to starting another period of PrEP).

7. Self-efficacy, self-determination, autonomy, empowerment.

8. Social harms (eg, coercion, violence (including intimate partner violence, violence from family members or community members, etc), psychosocial harm, selfharm, etc) and whether these harms were corrected/ had redress available.

\section{PICO question 2-continuation}

Should PrEP continuation be available from a pharmacist, without a prescription?

Population: individuals taking PrEP.

Intervention: PrEP access through a pharmacy without a prescription by a health worker.

Comparator: PrEP access by prescription from a health worker.

Outcomes:

1. Use of PrEP (continued use or an intermittent pattern of use related to risk exposure).

2. Correct use of PrEP (either daily or event-driven), including stopping and starting.

3. HIV acquisition/incidence.

4. Side effects, adverse events and clinical harms (renal disease, STI acquisition, STI treatment).

5. Uptake of regular HIV testing (1 month after initiation and 3 monthly thereafter while taking PrEP-or if taking PrEP intermittently (seasons of risk), prior to starting another period of PrEP).

6. Self-efficacy, self-determination, autonomy, empowerment.

7. Social harms (eg, coercion, violence (including intimate partner violence, violence from family members or community members, etc), psychosocial harm, selfharm, etc), and whether these harms were corrected/ had redress available.

\section{Inclusion criteria}

To be included in the effectiveness review for either PICO question, an article had to meet the following criteria:

1. Study design that compared PrEP access through a pharmacy without a prescription by a health worker to PrEP access by prescription from a health worker. This included randomised controlled trials (RCTs), non-RCTs and comparative observational studies (including prospective controlled cohort studies, crosssectional studies, controlled before-after studies and interrupted time series) that compared individuals who received the intervention with those who did not.

2. Measured one or more of the outcomes listed above. 
3. Published in a peer-reviewed journal or as a conference abstract.

If studies met all other criteria but did not present comparative data, we considered them 'case studies'. No restrictions were placed based on location of the intervention. No language restrictions were used on the search. Articles in English, French, Spanish and Chinese were coded directly; articles in other languages were translated.

\section{Search strategy}

The following electronic databases were searched through the search date of 2 December 2020: PubMed, CINAHL, LILACS and EMBASE. We searched for ongoing RCTs through clinicaltrials.gov, the WHO International Clinical Trials Registry Platform, the Pan-African Clinical Trials Registry and the Australian New Zealand Clinical Trials Registry. We searched abstracts from the following conferences: International AIDS Conference (AIDS), International AIDS Society Conference on HIV Science, International AIDS Society Conference on HIV Pathogenesis, Treatment and Prevention, HIV Research for Prevention and Retroviruses and Opportunistic Infections and HIV Research for Prevention. Only abstracts available electronically were included. Secondary reference searching was conducted on all studies included in the review. Finally, selected experts in the field were contacted to identify additional articles not identified through other search methods. The full search strategy for databases, registries and conference websites can be found in online supplemental appendix A.

\section{Screening abstracts}

Titles, abstracts, citation information and descriptor terms of citations identified through the search strategy were screened by a member of the senior study staff. Fulltext articles were obtained of all selected abstracts and two independent reviewers assessed all full-text articles for eligibility to determine final study selection. Differences were resolved through consensus.

\section{Data extraction and management}

Data were extracted independently by two reviewers using standardised data extraction forms. Differences in data extraction were resolved through consensus and referral to a senior study team member from $\mathrm{WHO}$ when necessary.

The coding form collected the following information from each included study:

- Study identification: author(s); type of citation; year of publication.

- Study description: study objectives; location; population characteristics; type of PrEP; PrEP initiation or continuation; study design; sample size; follow-up periods and loss to follow-up.

- Outcomes: analytic approach; outcome measures; comparison groups; effect sizes; CIs; significance levels; conclusions; limitations.
For RCTs, risk of bias was assessed using the Cochrane Collaboration's tool for assessing risk of bias. ${ }^{14}$ For nonRCTs but comparative studies, study rigour was assessed using the Evidence Project 8-item checklist for intervention evaluations. ${ }^{15}$

\section{Data analysis}

Data were analysed according to coding categories and outcomes. Where multiple studies reported the same comparative outcome, we planned to conduct metaanalysis using random-effects models to combine risk ratios with comprehensive meta-analysis.

We planned to stratify all PICO analyses by the following categories/subgroups (and intersections of these groups), where data were available:

- Type of PrEP (daily oral pill, event-driven, dapivirine vaginal ring, etc).

- Populations (eg, age, gender, race/ethnicity, key populations (men who have sex with men (MSM), sex workers, people who use drugs, transgender people, prisoners), etc).

- Vulnerabilities (ie, poverty, disability, literacy/educational level).

- High-income versus low-income or middle-income countries.

- Condom use.

We planned to summarise PICO findings in Grading of Recommendations, Assessment, Development and Evaluations (GRADE) Evidence Profile tables using GRADEPro. Case studies were summarised descriptively according to coding categories and outcomes.

\section{Values and preferences review}

The same search terms were used to search and screen for studies on the values and preferences of end users and health workers. Studies were included in this review if they presented primary data examining preferences of PrEP users, or individuals who might be or represent candidates for PrEP. We also included studies examining the values and preferences of health workers, including pharmacists and community health workers. From these populations, we sought studies examining opinions, perspectives, values and preferences related to PrEP access through pharmacies, or comparing PrEP access through pharmacies with other access points. We also considered issues related to age of availability, informed decision-making, coercion, seeking redress and stigma and discrimination (anticipated and experienced) in accessing PrEP through pharmacies. These studies could be qualitative or quantitative in nature, but had to present primary data collection-think pieces and review articles were not included. Values and preferences literature were summarised qualitatively and were organised by study design and methodology, location and population.

\section{Cost review}

The same search terms were used to search and screen for studies to be included in the cost review. Studies were 


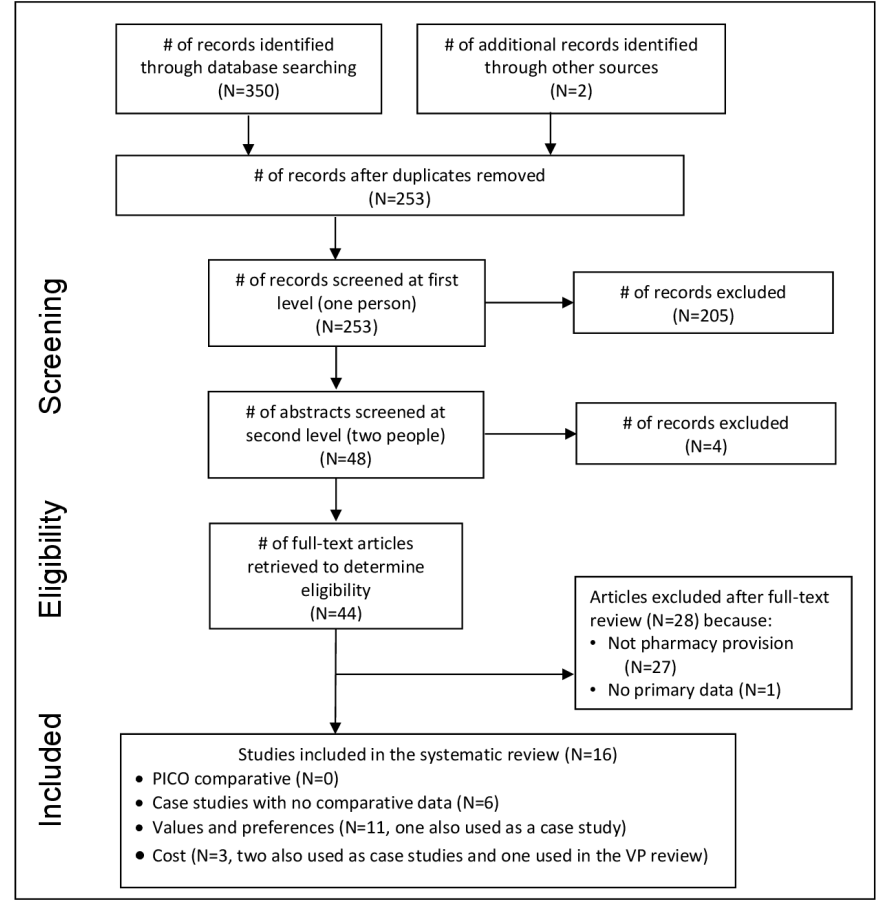

Figure 1 Preferred Reporting Items for Systematic Reviews and Meta-Analyses flow diagram showing disposition of citations through the search and screening process. PICO, population, intervention, comparison and outcomes.

included in this review if they presented primary data comparing costing, cost-effectiveness, cost-utility or costbenefit of PrEP initiation or continuation in pharmacies. Cost literature was summarised qualitatively. Cost literature was classified into four categories (health sector costs, other sector costs, patient/family costs and productivity impacts) and within each category was organised by study design/methodology, location and population.

\section{Patient and public involvement}

Feedback on the review protocol and analysis was received from the WHO patient safety working group. Patients were involved in a global survey of values and preferences conducted to inform the WHO guideline on selfcare interventions; they thus play a significant role in the overall recommendation informed by this review.

\section{RESULTS}

Our search strategy yielded 253 unique records, of which 16 were ultimately included in the systematic review (figure 1). Of these 17 studies, 0 were included in the effectiveness review but 6 were included as case studies, 11 were included in the values and preferences review and 3 were included in the cost review.

\section{Effectiveness review}

No articles met the inclusion criteria for the primary PICO questions, either PrEP initiation or continuation.

However, we did identify six 'case studies' where PrEP was offered through pharmacies, but where there was no data comparing this to provision by prescription only.
These were reported collectively in six articles and two abstracts. $^{16-23}$

Table 1 presents descriptive information about the six case studies. All six case studies were conducted in urban areas in the USA, although they came from diverse regions and served diverse populations. Most described operation through a collaborative practice agreement (CPA), where pharmacists operated under physician oversight. Most of the case studies described PrEP programmes that provided client counselling and risk assessment, lab testing and PrEP dispensing. In some cases, PrEP was initiated at the pharmacy and then patients had the option to continue elsewhere, while in other cases continuation occurred at the pharmacy.

Case studies provided descriptive data on the number of clients they served; some reported additional data on client demographics, test results and PrEP continuation. Where distribution of clients by sex and sexual orientation was reported, programmes said a majority of clients were male, and most were MSM. One study reported no differences in PrEP initiation or retention by client sex. ${ }^{16}$ Client race varied substantially by setting from $83.3 \%$ white $^{21}$ to $77 \%$ black $^{16}$ to $47 \%$ Hispanic/Latino. ${ }^{17}$ Insurance coverage varied from $35 \%^{16}$ to $80 \%^{21}$ of PrEP clients.

One large case study from Seattle enrolled 695 clients on $\mathrm{PrEP}^{20}$; the remaining case studies reported smaller PrEP enrollments of between 50 and 200 clients. Across studies, among clients who were referred for PrEP or completed a PrEP screening visit, between $74 \%{ }^{19} 20$ and $96 \%{ }^{17}$ started PrEP or filled their prescription, often on the same day or within a week.

Follow-up rates varied. In one study, $43 \%$ (23/53) of clients who filled their prescription attended their initial clinical appointment within 6 weeks of obtaining PrEP. ${ }^{16}$ The largest study reported a $25 \%$ drop-out rate and a mean duration of PrEP use of 302 days. $^{20}$

Two studies reported on HIV seroconversions among clients: one reported no seroconversions among PrEP clients, ${ }^{22} 23$ and the other reported no seroconversions among active clients but a seroconversion among a client who was lost to follow-up but then returned for PrEP and was diagnosed on HIV testing at the return visit. ${ }^{20}$ One study also reported HIV post-exposure prophylaxis (PEP), noting that six clients received PEP prior to initiation of PrEP. ${ }^{17}$

\section{Values and preferences review}

For the values and preferences review, 11 studies were identified, including one study that was also included in the case study review. ${ }^{21}{ }^{24-29}$ The majority $(n=8)$ were conducted in the USA, but two were conducted in Kenya, and one was conducted in South Africa. Seven used quantitative methods, generally cross-sectional surveys, while four used qualitative methods, generally in-depth interviews.

Table 2 presents descriptive data for the values and preferences studies, stratified by end users (including potential PrEP candidates, current PrEP users or general 
Table 1 Description of articles included in the case study review

\begin{tabular}{|c|c|c|c|}
\hline Study & Location & Description & Results \\
\hline Ryan et $a l^{2223}$ & $\begin{array}{l}\text { USA: } \\
\text { Albuquerque, } \\
\text { New Mexico }\end{array}$ & $\begin{array}{l}\text { One of the first pharmacy-run HIV PrEP clinics in the USA was established } \\
\text { in July } 2015 \text {. The half-day weekly clinic generally sees 10-14 patients per } \\
\text { week. Over } 200 \text { patients have been seen overall. }\end{array}$ & $\begin{array}{l}\text { There were no HIV seroconversions among those } \\
\text { who started PrEP. Of the first } 136 \text { clients, } 2 \text { tested } \\
\text { HIV-positive at baseline and } 127 \text { were started on PrEP } \\
\text { (TDF/FDC). One discontinued due to side effects. No } \\
\text { significant elevation in serum creatinine was noted } \\
\text { over time. Average adherence was }<1 \text { missed doses } \\
\text { per month and a median compliance rate of } 0.99 \text {. }\end{array}$ \\
\hline Havens et $a l^{21}$ & $\begin{array}{l}\text { USA: Omaha, } \\
\text { Nebraska }\end{array}$ & $\begin{array}{l}\text { P-PrEP allowed pharmacists to serve as PrEP providers through a CPA. } \\
\text { Pharmacists received education on HIV risk assessment, testing, risk } \\
\text { reduction counselling and administration of PrEP. Eligible participants } \\
\text { received a 90-day F/TDF prescription and had the option to continue PrEP } \\
\text { care at the university-based HIV clinic or at one of three participating sites } \\
\text { (community pharmacy, university-based primary care clinic or community } \\
\text { primary care clinic). Follow-up visits were every } 3 \text { months after PrEP } \\
\text { initiation, and laboratory monitoring was performed, including screening }\end{array}$ & $\begin{array}{l}60 \text { participants enrolled in the P-PrEP programme } \\
\text { and started F/TDF. The majority, } 91.7 \%(55 / 60) \text {, } \\
\text { were men, } 83.3 \% \text { (50/60) were white, } 80 \%(48 / 60) \\
\text { were commercially insured and } 89.8 \%(54 / 60) \text { had } \\
\text { completed some college or higher. The mean age of } \\
\text { participants was } 34 \text { years (range } 20-61 \text { years), and } \\
88.3 \%(53 / 60) \text { identified as MSM. }\end{array}$ \\
\hline
\end{tabular}
for HIV, chlamydia and gonorrhoea.

$\begin{array}{ll}\begin{array}{l}\text { Khosropour USA: Jackson, } \\ \text { et al }{ }^{16}\end{array} & \begin{array}{l}\text { The pharmacist evaluated patients for medical contraindications to PrEP, } \\ \text { but no baseline labs were obtained. The pharmacist provided a PrEP }\end{array} \\ & \begin{array}{l}\text { prescription and scheduled a clinical appointment for patients within } 6 \\ \text { weeks, at which time they were evaluated by a clinician and completed } \\ \text { baseline labs. }\end{array}\end{array}$

The pharmacist evaluated 69 patients for PrEP; $57 \%$ were MSM, $77 \%$ were black and $65 \%$ were uninsured. All patients received a PrEP prescription; $83 \%$ the same day and $97 \%$ within 5 days. Fiftythree $(77 \%)$ of 69 clients filled the prescription; $87 \%$ of whom filled it within 1 week. Only $23(43 \%)$ of 53 clients who filled their prescription attended their initial clinical appointment within 6 weeks. There were no differences in PrEP initiation or retention by patient sex/gender.

Lopez et al ${ }^{17} \quad$ USA: San A community pharmacy and the DPH developed a CPA that allowed

In the first year, 6 patients received PEP and 53

Francisco, community pharmacists to initiate PrEP and PEP. Pharmacists were trained California by DPH staff members on HIV testing and counselling and implementation of the PrEP protocol, including PEP initiation and STI testing. A DPH physician reviewed patients' charts regularly and communicated with PrEP pharmacists as needed.

\begin{tabular}{|c|c|c|c|}
\hline $\begin{array}{l}\text { Sawkin and } \\
\text { Shah }^{18}\end{array}$ & $\begin{array}{l}\text { USA: Kansas } \\
\text { City, Missouri }\end{array}$ & $\begin{array}{l}\text { Clinical pharmacists were trained to provide PrEP education and } \\
\text { medication management outlined within a CPA. The screening visit } \\
\text { includes rapid HIV testing, hepatitis C screening, urinalysis, pregnancy } \\
\text { testing, complete blood count with differential, comprehensive metabolic } \\
\text { profile, STI screening and hepatitis B serology. Once deemed eligible, } \\
\text { pharmacists prescribe TDF/FDC for up to } 90 \text { days to ensure medication } \\
\text { safety and efficacy. Patients return every } 3 \text { months for labs including rapid } \\
\text { HIV testing, a basic metabolic panel and STD screening. }\end{array}$ & $\begin{array}{l}\text { In the first year, the PrEP clinic had }>50 \text { actively } \\
\text { managed patients. }\end{array}$ \\
\hline Tung et $a l^{1920}$ & $\begin{array}{l}\text { USA: Seattle, } \\
\text { Washington } \\
\text { state }\end{array}$ & $\begin{array}{l}\text { The One-Step PrEP clinic, at a private pharmacy and under physician } \\
\text { oversight (1 first year resident physician, } 3 \text { pharmacists, ancillary staff), } \\
\text { provides PrEP with a single patient encounter. Pharmacists meet with } \\
\text { patients individually, take a medical and sexual history, make a risk } \\
\text { assessment, perform laboratory testing, provide patient education and } \\
\text { prescribe and dispense oral PrEP (TDF/FTC) when appropriate. }\end{array}$ & $\begin{array}{l}\text { Of } 714 \text { patients evaluated, } 695 \text { ( } 97.3 \%) \text { initiated PrEP. } \\
\text { Mean duration of PrEP use was } 302 \text { days. Same-day } \\
\text { medication start: } 513 \text { ( } 74 \%) \text {. Drop-out rate: } 25 \% \text {. STI } \\
\text { diagnoses: } 207 \text { in } 135 \text { patients. HIV diagnoses: } 2 \text { at } \\
\text { initial evaluation, } 0 \text { during active engagement, } 1 \text { after } \\
\text { being lost to follow up. }\end{array}$ \\
\hline
\end{tabular}

CPA, collaborative practice agreement; DPH, Department of Public Health; MSM, men who have sex with men; P-PrEP, pharmacist-led PrEP; PrEP, pre-exposure prophylaxis; STI, sexually transmitted infection.

populations), or pharmacists, health workers and other professional stakeholders. Two studies included both potential end users and health workers. Table 3 presents findings from the values and preferences studies. Six studies from the USA, Kenya and South Africa found potential PrEP clients generally supported PrEP prescriptions in pharmacies, although some preferred clinics. For example, a discrete choice experiment focused on longacting PrEP options among youth in South Africa noted that location of PrEP access was relatively less important than other attributes such as dosing frequency, pain or insertion site, but that different populations expressed different location preferences: women preferred health clinic access, men who have sex with women only preferred community locations and MSM preferred pharmacy or health clinics. ${ }^{28}$ One study of current PrEP pharmacy users found all would 'definitely recommend' the programme. ${ }^{21}$ Six studies found pharmacists were generally supportive of offering $\operatorname{PrEP}^{21} 26$ 30-33; one study including doctors found less support, and one study of diverse Kenyan stakeholders found broad support. ${ }^{33}$ Benefits of pharmacy access included convenience, accessibility and alignment with scope of work. Concerns included inadequate time, compensation for services, privacy and training.

\section{Cost review}

Two of the case studies presented data about health sector costs and patient/family costs, ${ }^{19-21}$ and one values and preferences article also examined willingness to pay for PrEP. ${ }^{24}$ No studies looked at other sector costs or productivity impacts. Table 4 summarises the three studies included in the costs review. Both case studies which presented cost data were conducted in the USA. 
Table 2 Descriptions of values and preferences studies

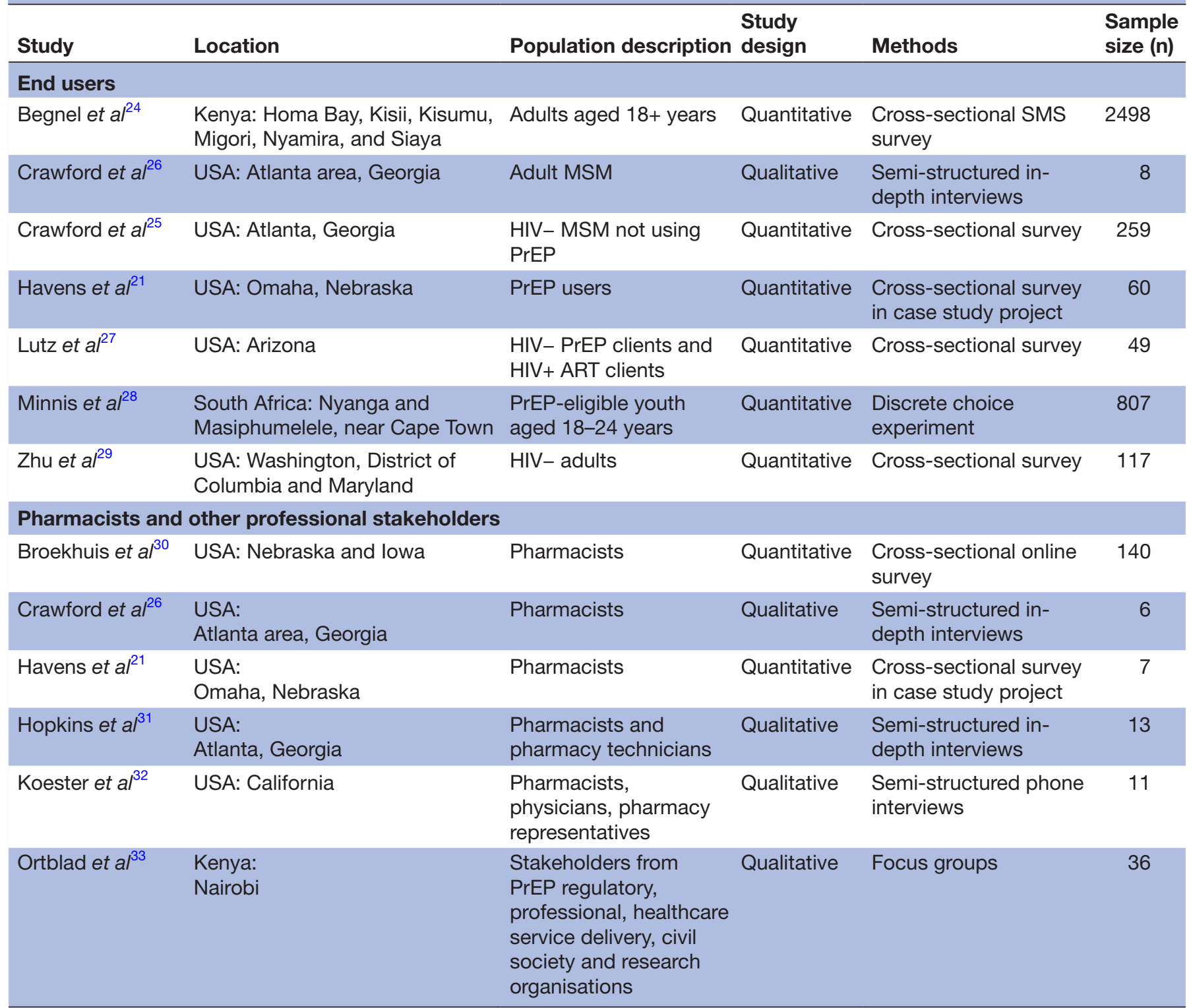

ART, antiretroviral therapy; MSM, men who have sex with men; PrEP, pre-exposure prophylaxis.

For health sector costs, one clinic reported it recouped start-up costs in 9 months, and financial sustainability was dependent on the ability of pharmacists to bill insurance plans for their services. ${ }^{19}{ }^{20}$ For patient/family costs, $98 \%$ of patients paid US\$0 for their PrEP in one study, and in another, participants were split in willingness to pay US $\$ 20$ or US $\$ 60$ quarterly for PrEP visits. ${ }^{19} 20$ Finally, one article from Kenya found over half of participants were willing to pay for PrEP and $78 \%$ said the maximum they would pay for a month's supply was $<\mathrm{US} \$ 5$. $^{24}$

\section{DISCUSSION}

This systematic review identified no studies for our primary PICO questions, indicating a paucity of evidence investigating the comparative effectiveness of pharmacyaccess versus provider-access to PrEP, for individuals initiating or continuing PrEP. However, we did identify six non-comparative case studies which provide some limited evidence on the feasibility of pharmacy distribution of PrEP. Although all were from the USA, all found pharmacy-access PrEP to be a feasible service delivery model.

The evidence base identified in our review was largely focused on the USA, with just three values and preferences studies and one cost study from sub-Saharan African settings. This represents a critical gap in the literature given global differences in pharmacy regulation and capacity, particularly in many settings with high HIV prevalence. Pharmacies in the USA are subject to substantial regulations, and pharmacists generally receive high levels of training and oversight, which may enable provision of high-quality services for PrEP through pharmacies. In 
Table 3 Key findings from values and preferences studies

\begin{tabular}{lll} 
Study & Location & Results \\
\hline $\begin{array}{l}\text { End users } \\
\text { Begnel et } \mathrm{al}^{24}\end{array}$ & Kenya & $\begin{array}{l}\text { When asked whether someone would be most likely to obtain PrEP at a clinic, pharmacy, kiosk or other location, } \\
44 \% \text { chose clinics, 37\% chose pharmacies, 17\% chose kiosks and 1\% chose other. }\end{array}$
\end{tabular}

Crawford et $a^{25}$ USA Most participants (69\%) were willing to discuss PrEP with pharmacy staff and $61.35 \%$ were willing to be screened for PrEP in pharmacy. There were no differences by race, after accounting for PrEP interest.

Crawford et $a^{26}$ USA Most MSM supported in-pharmacy STI, HIV and PrEP screenings and dissemination. Benefits included convenience and accessibility. Participants wanted to ensure privacy, confidentiality and welcoming staff for MSM.

\begin{tabular}{lll} 
Havens et al ${ }^{21}$ & USA & $\begin{array}{l}\text { At 6-month follow-up, all of the survey respondents stated they would definitely recommend the P-PrEP } \\
\text { programme. }\end{array}$ \\
\hline Lutz et al ${ }^{27}$ & USA & $\begin{array}{l}\text { 93.9\% were comfortable seeing a pharmacist to discuss PrEP, and 93.9\% were comfortable having a pharmacist } \\
\text { test for HIV before starting PrEP; } 83.7 \% \text { were comfortable having a pharmacist prescribe PrEP, although only four } \\
\text { participants (8.2\%) strongly agreed. }\end{array}$
\end{tabular}

Minnis et al ${ }^{28} \quad$ South In this discrete choice experiment about hypothetical long-acting PrEP options, 'where PrEP is available' was Africa relatively less important than other attributes such as dosing frequency, pain or injection site. Females preferred using a product that was offered at a health clinic over accessing it at a pharmacy $(p<0.001)$. Among males, men who have sex with women only had somewhat more preference for availability at a community location compared with a pharmacy and health clinic, whereas MSM held opposite views with pharmacy or health clinic preferred over a community location $(\mathrm{p}=0.01)$.

Zhu et $a^{29} \quad$ USA Most participants supported pharmacists prescribing PrEP (mean 4.0 (SD=1.0), range 3.9-4.1 on a scale of 1-5 with 5 strongly agree). Most (58.1\%) had no concerns; the most common concerns were 'prefer to obtain a prescription from my doctor' (16.2\%) and 'privacy concerns' (15.4\%). Participants were more likely to support pharmacy PrEP if they had previous interactions with pharmacists or if they had previously used PrEP (vs nonusers).

\begin{tabular}{|c|c|c|}
\hline \multicolumn{3}{|c|}{ Pharmacists and other professional stakeholders } \\
\hline $\begin{array}{l}\text { Broekhuis et } \\
a l^{30}\end{array}$ & USA & $\begin{array}{l}\text { Respondents were 'moderately concerned' or 'very concerned' about the following issues: time burden }(61 \%) \text {, } \\
\text { inadequate compensation for services }(55 \%) \text {, outside skill set }(39 \%) \text {, patient adherence to therapy }(63 \%) \text {, loss to } \\
\text { follow-up (56\%) and promotion of antiretroviral drug resistance }(51 \%) \text {. }\end{array}$ \\
\hline Havens et $a l^{21}$ & USA & $\begin{array}{l}\text { The P-PrEP pharmacists felt comfortable performing point-of-care testing at all visits except on one occasion } \\
(0.7 \%, 1 \text { of } 139) \text {. One pharmacist at the community pharmacy site reported three occasions }(2.2 \%) \text { in which } \\
\text { they felt uncomfortable conducting sexual histories during P-PrEP follow-up visits. Workflow disruption at the } \\
\text { community pharmacy site was reported only once }(0.7 \%) \text { throughout the study. }\end{array}$ \\
\hline Hopkins et $\left.a\right|^{31}$ & USA & $\begin{array}{l}\text { Pharmacists and pharmacy technicians expressed strong willingness and support for screening and dispensing } \\
\text { PrEP in pharmacies. Both groups expressed concerns about the time and the resources needed to perform PrEP } \\
\text { screening and dispensing. Technicians also reported concerns about privacy for patients as well as the need } \\
\text { for community support and awareness of pharmacy-based PrEP screening, and they recommended scheduling } \\
\text { of PrEP screening activities during a limited part of the day to facilitate screening. Pharmacists reported } \\
\text { fewer barriers but a need for more training of pharmacy staff to assist with PrEP screening and dispensing } \\
\text { implementation. }\end{array}$ \\
\hline Ortblad et $a l^{33}$ & Kenya & $\begin{array}{l}\text { Stakeholders were enthusiastic about a model for pharmacy-based PrEP delivery. Potential challenges identified } \\
\text { included insufficient pharmacy provider knowledge and skills, regulatory hurdles to providing affordable HIV } \\
\text { testing at pharmacies and undefined pathways for PrEP procurement. Potential solutions included having } \\
\text { pharmacy providers complete the Kenya Ministry of Health-approved PrEP training, use of a PrEP prescribing } \\
\text { checklist with remote clinician oversight and provider-assisted HIV self-testing and having the government provide } \\
\text { PrEP and HIV self-testing kits to pharmacies during a pilot test. }\end{array}$ \\
\hline
\end{tabular}

MSM, men who have sex with men; P-PrEP, pharmacist-led PrEP; PrEP, pre-exposure prophylaxis; STI, sexually transmitted infection.

other settings, training and regulation may be more variable, making quality control more challenging. Notably, most of the case studies specifically described using CPAs which require physician oversight of pharmacist provision of PrEP. Training may also vary significantly by type of health worker, as pharmacists (compared with nurses or physicians) typically receive more robust training in pharmacotherapy as well as monitoring for efficacy, toxicity and safety; the team-based approach to pharmacy distribution of PrEP may synergize the strengths of each type 


\begin{tabular}{|c|c|c|}
\hline Study & Location & Results \\
\hline Begnel et $a l^{24}$ & Kenya & $\begin{array}{l}\text { Over half }(61 \%) \text { of participants were willing to pay for PrEP and } 78 \% \text { reported that the maximum amount they } \\
\text { were willing to pay for a } 1 \text { month supply was }<\text { US } \$ 5 \text {. }\end{array}$ \\
\hline Havens et $a l^{21}$ & $\begin{array}{l}\text { USA: Omaha, } \\
\text { Nebraska }\end{array}$ & $\begin{array}{l}\text { Among participants who completed follow-up visits at the community pharmacy, half }(6 \text { of } 12) \text { stated they } \\
\text { would be willing to pay at least US } \$ 20 \text { quarterly for continued PrEP visits and half ( } 6 \text { of } 12) \text { were willing to pay } \\
\text { up to US } \$ 60 \text { quarterly. }\end{array}$ \\
\hline Tung et $a l^{1920}$ & $\begin{array}{l}\text { USA: Seattle, } \\
\text { Washington } \\
\text { state }\end{array}$ & $\begin{array}{l}\text { In the } 2017 \text { abstract, } 96 \% \text { of patients }(235 / 245) \text { paid US } \$ 0 \text { for their PrEP. Initial startup costs were recouped } \\
\text { after } 9 \text { months of operations. In the } 2018 \text { article, } 98 \% \text { of patients paid US } \$ 0 \text { for their PrEP (total } n=695) \text {. } \\
\text { Financial sustainability of the model was dependent on the ability of pharmacists to bill insurance plans for } \\
\text { their services in accordance with local legislative changes requiring commercial insurances to recognise } \\
\text { pharmacists as providers. }\end{array}$ \\
\hline
\end{tabular}

PrEP, pre-exposure prophylaxis.

of health worker. Most of the study pharmacies either had laboratory capacity or were well-connected with laboratories, providing an avenue for baseline tests and ongoing monitoring needed for PrEP. Where links with laboratories do not exist, it will be important to consider how they might be created to ensure appropriate support for PrEP initiation and continuation. ${ }^{7}$ In some settings, health systems have developed simplified laboratory testing for PrEP delivery, for example, by waiving creatinine testing, or have allowed HIV self-testing for PrEP continuation. However, these models were found to be highly feasible with few adverse outcomes, warranting further research in a wider range of settings.

In terms of values and preferences, we found that actual or potential PrEP clients were generally supportive of pharmacy-access PrEP. Many included studies did not describe in-depth reasons for or against pharmacy PrEP. In the USA, MSM emphasised the importance of privacy, confidentiality and having welcoming staff. ${ }^{21} 2629$ One study from South Africa highlighted the role of subgroup differences, finding that preferences for potential longacting PrEP differed between women, MSM and men who have sex with women. ${ }^{28}$ These differences align with previous findings about user preferences for PrEP delivery more broadly. ${ }^{34}$ Furthermore, even within subpopulations (eg, women), heterogeneity is to be expected as user preferences may be shaped by geographic, economic and sociocultural contexts. ${ }^{36}$

In particular, we found that pharmacy delivery of PrEP was highly accepted among marginalised groups, such as black MSM in the south of the USA. ${ }^{16} 25$ As these groups face critical barriers to accessing PrEP through more traditional modalities, pharmacy PrEP may be an important additional option for them. ${ }^{37}$ Understanding the perspectives of other groups often excluded from research on PrEP users' values and preferences, such as transgender people, sex workers or people who use drugs, is also critical. ${ }^{34}$

Evidence from health workers indicated mixed support for pharmacy-access PrEP. Some had concerns about the added time associated with a new task, although one of our included case studies found that workflow disruption was minimal. ${ }^{21}$ Concerns about insufficient training and skills to provide PrEP were common. ${ }^{30-33}$ While guidelines and clinical requirements at PrEP visits vary across settings, ${ }^{38}$ pharmacists require training and supervision to provide HIV and creatinine clearance testing at a minimum, along with pregnancy testing, STI screening and other diagnostic tests depending on setting and population. Training may also be required regarding other aspects of integrated SRH, such as contraceptive provision or referral for people at risk of violence. For instance, lockdowns during the COVID-19 pandemic have limited access to health services, and task sharing to pharmacists can support a range of health interventions. The WHO Academy ${ }^{10}$ module on counselling and prescribing of contraception in pharmacies has developed competency-based learning for pharmacists which could be further extended to other health areas, including provision of PrEP. Along with training and supervision, strategies to support laboratory access-whether on-site or elsewherewill be key to offering PrEP through pharmacies. This might require changes to supply chain systems to ensure uninterrupted supply of PrEP medications for pharmacies, as well as changes to health management information systems to ensure that pharmacy-level activities can be captured. Furthermore, monitoring of pharmacy-access PrEP would have to become part of routine monitoring efforts in order to help ensure quality service provision.

Two of our included studies assessed client willingness to pay for pharmacy-access PrEP. Willingness to pay ranged from US\$5 per month in Kenya ${ }^{24}$ to as much as US\$20 per month in the USA. ${ }^{21}$ In terms of overall financial sustainability of the PrEP pharmacy model, in the USA, one study found this was achievable but entirely dependent on insurance billing. Since costs and willingness to pay will vary substantially by income, setting and health system or insurance/reimbursement structure, further research in this area is needed. Additional evidence could elucidate cost differences in countries where national health insurance programmes partner with pharmacies, compared with those where government services are generally provided for free or at low cost. Broadly, given the high HIV burden and rapid scale-up of PrEP in subSaharan Africa, ${ }^{39} 40$ more costing data from this region are needed. For example, some African PrEP programmes have adopted models wherein service delivery costs are shared across interventions through shared service platforms. ${ }^{41-43}$ It 
may be that integrating PrEP into pharmacies offers similar opportunities for cost-sharing across programmes and interventions $^{44}$; this warrants further exploration.

While the studies included in our review all focused on daily oral PrEP except for one hypothetical values and preferences study on long-acting injectable PrEP, the monthly dapivirine vaginal ring is included in the WHO list of prequalified products and in recent $\mathrm{WHO}$ guidelines ${ }^{45}$ as an additional prevention choice for women. Its high safety profile and low systemic absorption reduce requirements for laboratory monitoring may make pharmacy delivery and option for established women users. Long-acting injectable PrEP (cabotegravir), which is given by intramuscular injection every 8 weeks, is likely to gain regulatory approval and could potentially be considered in the future for pharmacy provision, if complexities with HIV testing and other implementation issues are resolved. Long-acting injectable PrEP formulations have been viewed favourably by potential end users. ${ }^{46}$ Future studies should consider the safety and effectiveness of delivering long-acting PrEP products such as the dapivirine vaginal ring and long-acting injectable PrEP at pharmacies, and end user and health worker preferences around this delivery option. These interventions might also be considered as part of a broader package of SRH-related services that could be managed to the pharmacy-level, which might help maximise efficiencies and minimise stigma associated with standalone HIV interventions.

Our review had several strengths and limitations. We conducted a comprehensive search for articles on effectiveness, and on values and preferences of end users and health workers, as well as cost data. However, our focus on peer-reviewed articles and conference abstracts may have missed some relevant information from programme reports or other grey literature. We missed the words 'initiation', 'initiate' and 'initiated' in our search terms, but believe that most articles describing pharmacist initiation of PrEP would have used either 'pharmacy' or 'pharmacist' so would have been captured by our search. We also acknowledge that this is a new and rapidly growing field; we may have excluded articles which would have met our inclusion criteria but were published after our search date, including at least one acceptability and feasibility study conducted among clients and health workers in Kenya which reinforced our findings of support for expanding PrEP to retail pharmacies, although participants wanted to ensure that such services would be 'private, respectful, safe and affordable'. ${ }^{47}$ Our conclusions are also limited because nearly all the evidence identified in our review came from the USA, except for a few studies from Kenya and South Africa. Future research should continue to examine the potential for pharmacy provision of PrEP in resource-limited settings.

\section{CONCLUSIONS}

Overall, we found that while pharmacy distribution of PrEP has been shown to be feasible in some studies in the USA and valued by end users in small studies, there is a lack of evidence around its effectiveness or its adaptability to low-income and middle-income settings. As PrEP services continue to expand worldwide, additional research and programmatic efforts into pharmacy delivery are warranted. The services, staffing, infrastructure and regulation of pharmacies varies considerably between and within countries; if PrEP products are to be delivered through these settings, minimum service requirements and staff training needs will need to be considered. With the increasing roll-out of PrEP across regions, more evidence from safety monitoring may reduce laboratory monitoring requirements, and the COVID-19 pandemic has led to adaptations to support continuation of PrEP delivery such as the use of HIV self-testing, virtual platforms and telemedicine support. Future implementation research could explore how these could be incorporated into future PrEP pharmacy models. This evidence base should be informed by variation across contexts, screening and laboratory requirements, and values and preferences of affected populations and health workers. Privacy, confidentiality and quality of services will be important to ensure for all clients. Overall, pharmacy access may be a promising strategy for expanding access to PrEP, improving equity and helping to respect, protect and fulfil the right to health.

Acknowledgements We thank Johns Hopkins graduate students Hunied Kautsar, Jingjia (Cynthia) Li and Xuhao Yang for their help screening citations and coding articles for this review. We also thank Shona Dalal, Robin Schaefer, Michelle Rodolph and Nandi Siegfried for their thoughtful comments on the protocol and/or draft manuscript.

Contributors MN conceptualised the study, following discussion with RB. CEK and PTY designed the protocol, with feedback from MN, RB and LF. PTY ran the database search and oversaw the search, screening, full-text review and data extraction process. CEK and KA drafted the manuscript. All authors reviewed the draft, provided critical review and read and approved the final manuscript. The corresponding author, as guarantor, accepts full responsibility for the finished article has access to any data and controlled the decision to publish. The corresponding author attests that all listed authors meet the authorship criteria and that no others meeting the criteria have been omitted. The named authors alone are responsible for the views expressed in this publication and do not necessarily represent the decisions or the policies of the World Health Organization (WHO) or the UNDPUNFPA-UNICEF-WHO-World Bank Special Programme of Research, Development and Research Training in Human Reproduction (HRP).

Funding This study was funded by The Children's Investment Fund Foundation. All authors had full access to all of the data in the study and can take responsibility for the integrity of the data and the accuracy of the data analysis.

Disclaimer The funder played no part in the decision to submit the article for publication, nor in the collection, analysis and interpretation of data.

Competing interests None declared.

Patient consent for publication Not applicable.

Ethics approval Ethical approval was not required for this systematic review, since all data came from information freely available in the public domain (ie, published articles or conference abstracts). This study does not involve human participants.

Provenance and peer review Not commissioned; externally peer reviewed.

Data availability statement Data are available on reasonable request. Extracted data are available on request to the corresponding author.

Supplemental material This content has been supplied by the author(s). It has not been vetted by BMJ Publishing Group Limited (BMJ) and may not have been peer-reviewed. Any opinions or recommendations discussed are solely those of the author(s) and are not endorsed by BMJ. BMJ disclaims all liability and responsibility arising from any reliance placed on the content. Where the content 
includes any translated material, BMJ does not warrant the accuracy and reliability of the translations (including but not limited to local regulations, clinical guidelines, terminology, drug names and drug dosages), and is not responsible for any error and/or omissions arising from translation and adaptation or otherwise.

Open access This is an open access article distributed in accordance with the Creative Commons Attribution Non Commercial (CC BY-NC 4.0) license, which permits others to distribute, remix, adapt, build upon this work non-commercially, and license their derivative works on different terms, provided the original work is properly cited, appropriate credit is given, any changes made indicated, and the use is non-commercial. See: http://creativecommons.org/licenses/by-nc/4.0/.

\section{ORCID iD}

Ping Teresa Yeh http://orcid.org/0000-0002-7425-0382

\section{REFERENCES}

1 WHO. Guidelines: updated recommendations on HIV prevention, infant diagnosis, antiretroviral initiation and monitoring. Geneva: World Health Organization, 2021.

2 WHO. Preventing HIV and other STIs among women and girls using contraceptive services in contexts with high HIV incidence: actions for improved clinical and prevention services and choices, 2020. Available: https://www.who.int/publications/i/item/actions-forimproved-clinical-and-prevention-services-and-choices-preventinghiv-and-other-stis-among-women-and-girls-using-contraceptiveservices

3 Mayer $\mathrm{KH}$, Chan PA, R Patel R, et al. Evolving models and ongoing challenges for HIV preexposure prophylaxis implementation in the United States. J Acquir Immune Defic Syndr 2018;77:119-27.

4 Farley JE, Dangerfield DT, LaRicci J, et al. Community engagement and linkage to care efforts by peer community-health workers to increase PreP uptake among sexual minority men. Public Health Nurs 2021;38:818-24.

5 Touger R, Wood BR. A review of telehealth innovations for HIV preexposure prophylaxis (PreP). Curr HIVIAIDS Rep 2019;16:113-9.

6 Myers JE, Farhat D, Guzman A, et al. Pharmacists in HIV prevention: an Untapped potential. Am J Public Health 2019;109:859-61.

7 Lopez MI, Grant RM, Dong BJ. Community pharmacy delivered PreP to stop HIV transmission: an opportunity not to miss! J Am Pharm Assoc 2020;60:e18-24.

8 Farmer EK, Koren DE, Cha A, et al. The pharmacist's expanding role in HIV pre-exposure prophylaxis. AIDS Patient Care STDS 2019;33:207-13.

9 WHO. WHO consolidated guideline on self-care interventions for health: sexual and reproductive health and rights. Geneva, Switzerland: World Health Organization, 2019. https://www.who.int/ reproductivehealth/publications/self-care-interventions/en/

10 WHO. WHO Academy, 2021. Available: https://www.who.int/about/ who-academy

11 WHO. Maintaining essential health services: operational guidance for the COVID-19 context interim guidance. Geneva, Switzerland: World Health Organization, 2020. https://www.who.int/publications/i/item/ WHO-2019-nCoV-essential-health-services-2020.1

12 WHO. Maintaining essential health services: operational guidance for the COVID-19 context interim guidance, 2020. Available: https:// www.who.int/publications/i/item/WHO-2019-nCoV-essential-healthservices-2020.1

13 Moher D, Liberati A, Tetzlaff J, et al. Preferred reporting items for systematic reviews and meta-analyses: the PRISMA statement. PLoS Med 2009;6:e1000097.

14 Higgins JPT, Thomas J, Chandler J. Cochrane Handbook for systematic reviews of interventions, 2019.

15 Kennedy CE, Fonner VA, Armstrong KA, et al. The evidence project risk of bias tool: assessing study rigor for both randomized and nonrandomized intervention studies. Syst Rev 2019;8:3.

16 Khosropour CM, Backus KV, Means AR, et al. A pharmacist-led, same-day, HIV pre-exposure prophylaxis initiation program to increase PreP uptake and decrease time to PreP initiation. AIDS Patient Care STDS 2020;34:1-6.

17 Lopez MI, Cocohoba J, Cohen SE, et al. Implementation of pre-exposure prophylaxis at a community pharmacy through a collaborative practice agreement with San Francisco department of public health. J Am Pharm Assoc 2020;60:138-44.

18 Sawkin M, Shah M. Development of a pharmacist-led human immunodeficiency virus pre-exposure prophylaxis clinic at an urban community clinic. Journal of the American Pharmacists Association 2016;56:e48.
19 Tung E, Thomas A, Eichner A. Feasibility of a pharmacist-run HIV PreP clinic in a community pharmacy setting. Topics in Antiviral Medicine 2017;25:407s.

20 Tung EL, Thomas A, Eichner A, et al. Implementation of a community pharmacy-based pre-exposure prophylaxis service: a novel model for pre-exposure prophylaxis care. Sex Health 2018;15:556-61.

21 Havens JP, Scarsi KK, Sayles H, et al. Acceptability and feasibility of a pharmacist-led HIV pre-exposure prophylaxis (PreP) program in the midwestern United States. Open Forum Infect Dis 2019;6.

22 Ryan K, Lewis J, Sanchez D, et al. 1293. the next step in PreP: evaluating outcomes of a Pharmacist-Run HIV pre-exposure prophylaxis (PreP) clinic. Open Forum Infect Dis 2018;5:S395.

23 Ryan KL, Jakeman B, Conklin J, et al. Treatment of patients with HIV or hepatitis $\mathrm{C}$ by pharmacist clinicians in a patient-centered medical home. Am J Health Syst Pharm 2019;76:821-8.

24 Begnel ER, Escudero J, Mugambi M, et al. High pre-exposure prophylaxis awareness and willingness to pay for pre-exposure prophylaxis among young adults in Western Kenya: results from a population-based survey. Int J STD AIDS 2020;31:454-9.

25 Crawford ND, Albarran T, Chamberlain A, et al. Willingness to discuss and screen for pre-exposure prophylaxis in pharmacies among men who have sex with men. J Pharm Pract 2021;34:734-40.

26 Crawford ND, Josma D, Morris J, et al. Pharmacy-based preexposure prophylaxis support among pharmacists and men who have sex with men. J Am Pharm Assoc 2020;60:602-8.

27 Lutz S, Heberling M, Goodlet KJ. Patient perspectives of pharmacists prescribing HIV pre-exposure prophylaxis: a survey of patients receiving antiretroviral therapy. J Am Pharm Assoc 2021;61:e75-e79.

28 Minnis AM, Atujuna M, Browne EN, et al. Preferences for long-acting Pre-Exposure prophylaxis (PreP) for HIV prevention among South African youth: results of a discrete choice experiment. J Int AIDS Soc 2020;23:e25528.

29 Zhu V, Tran D, Banjo O, et al. Patient perception of community pharmacists prescribing pre-exposure prophylaxis for HIV prevention. J Am Pharm Assoc 2020;60:781-8.

30 Broekhuis JM, Scarsi KK, Sayles HR, et al. Midwest pharmacists familiarity, experience, and willingness to provide pre-exposure prophylaxis (PreP) for HIV. PLoS One 2018;13:e0207372.

31 Hopkins R, Josma D, Morris J, et al. Support and perceived barriers to implementing pre-exposure prophylaxis screening and dispensing in pharmacies: examining concordance between pharmacy technicians and pharmacists. J Am Pharm Assoc 2021;61:115-120.

32 Koester KA, Saberi P, Fuller SM, et al. Attitudes about community pharmacy access to HIV prevention medications in California. J Am Pharm Assoc 2020;60:e179-83.

33 Ortblad KF, Mogere P, Roche S, et al. Design of a care pathway for pharmacy-based PreP delivery in Kenya: results from a collaborative stakeholder consultation. BMC Health Serv Res 2020;20:1034.

34 Eakle R, Weatherburn P, Bourne A. Understanding user perspectives of and preferences for oral PreP for HIV prevention in the context of intervention scale-up: a synthesis of evidence from sub-Saharan Africa. J Int AIDS Soc 2019;22 Suppl 4:e25306.

35 Koechlin FM, Fonner VA, Dalglish SL, et al. Values and preferences on the use of oral pre-exposure prophylaxis (PreP) for HIV prevention among multiple populations: a systematic review of the literature. AIDS Behav 2017;21:1325-35

36 Minnis AM, Browne EN, Boeri M, et al. Young women's stated preferences for biomedical HIV prevention: results of a discrete choice experiment in Kenya and South Africa. J Acquir Immune Defic Syndr 2019;80:394-403.

37 Edeza A, Karina Santamaria E, Valente PK, et al. Experienced barriers to adherence to pre-exposure prophylaxis for HIV prevention among MSM: a systematic review and meta-ethnography of qualitative studies. AIDS Care 2021;33:1-9.

38 WHO. Guideline on when to start antiretroviral therapy and on pre-exposure prophylaxis for HIV. Geneva, Switzerland: World Health Organization, 2015. https://www.who.int/hiv/pub/guidelines/ earlyrelease-arv/en/

39 Ahmed N, Pike C, Bekker L-G. Scaling up pre-exposure prophylaxis in sub-Saharan Africa. Curr Opin Infect Dis 2019;32:24-30.

40 Irungu EM, Baeten JM. Prep rollout in Africa: status and opportunity. Nat Med 2020;26:655-64.

41 South Africa National Department of Health. Guidelines for the provision of pre-exposure prophylaxis (PreP) to persons at substantial risk of HIV infection 2020.

42 National AIDS \& STI Control Program. Framework for the implementation of pre-exposure prophylaxis of HIV in Kenya. Kenya Ministry of health, 2017. Available: https://prep.nascop.org/policiesand-guidelines/ 
43 Zimbabwe Ministry of Health and Child Care. Implementation plan for HIV pre-exposure prophylaxis in Zimbabwe, 2017. Available: http:// www.mohcc.gov.zw/index.php?option=com_content\&view=article\& id $=189 \&$ ltemid $=732$

44 Roberts DA, Barnabas RV, Abuna F, et al. The role of costing in the introduction and scale-up of HIV pre-exposure prophylaxis: evidence from integrating PreP into routine maternal and child health and family planning clinics in Western Kenya. J Int AIDS Soc 2019;22:e25296.
45 WHO. Updated recommendations on HIV prevention, infant diagnosis, antiretroviral initiation and monitoring. WHO, 2021.

46 Clement ME, Kofron R, Landovitz RJ. Long-Acting injectable cabotegravir for the prevention of HIV infection. Curr Opin HIV AIDS 2020;15:19-26.

47 Roche SD, Wairimu N, Mogere P, et al. Acceptability and feasibility of Pharmacy-Based delivery of pre-exposure prophylaxis in Kenya: a qualitative study of client and provider perspectives. AIDS Behav 2021;25:3871-82. 